\title{
A Patient Treatment System Using RFID and Internet Communication
}

\author{
Heung-Kuk Jo, Member, KIMICS
}

\begin{abstract}
Medical technology is gradually being developed by applying information technologies. Especially, RFID technology is being used for precise disease history information of patients [4]. And in case the patient is far away, the patient can be treated using network communication of the internet [5][6]. The internet makes us to treat or operate the patient without being restricted to time or space. If the above technologies are made as a system, the patient can be treated or operated without being restricted to time or space. In this paper, we present a patient treatment system has been implemented with a system using RFID and network communication of the internet [1][2][3][4]. The system is driven as follows. First, the information of patient can be checked from a remote $\mathrm{PC}$, if the tag that a patient has been read through a reader. And a remote treatment is performed by controlling robot's arm with a joystick using internet network $[19][20][21]$. The RFID system was implemented in frequency of $125 \mathrm{KHz}$ [1]. The information of patient can be checked with PDA, $P C$ and C-LCD using Bluetooth and WLAN [7][8][9][10]. For the treatment and operation of the patient, the robot's arm has been formed using AX-12 motor, joystick and two buttons [11][12][13][14][15] [17][18].
\end{abstract}

Keywords - Treatment, RFID, Internet, Bluetooth, WLAN

\section{INTRODUCTION}

The most widely used recognition technology of patient in the modern society is the RFID technology[1][2]. This technology has high recognition rate and can be easily attached to the subject as the shape of tag is very diverse. Generally, the tag for patients is in a form of watch. The internet network is used for remote treatment. The advantage of this network is that the treatment or operation can be performed without being restricted to time or place. The communication method uses TCP/IP method and the system can be implemented easily [10].

In this paper, a system enabling verification of patient information and treatment has been implemented using RFID and Ethernet. Once the patient's tag is verified from a PC through $125 \mathrm{KHz}$ reader, the tag information of patient get sent up to remote distance through TCP/IP communication. Through this process, the name of patient and name of disease, etc can be verified. After checking the

\footnotetext{
Manuscript received August 20, 2010; revised September 8, 2010; accepted September 12, 2010.

Heung-Kuk Jo is with the Engineering of Computer Information Communication, Dong-Seo University, Busan, 617-716, Korea (Email: hkjo@gdsu.dongseo.ac.kr)
}

information of patient through $\mathrm{PC}$, the robot's arm is controlled from a remote distance using internet network through a joystick for the treatment and operation of patient. As a conclusion, the photos of each system parts of the system are shown and the response time on each communication process is shown as table.

\section{SYSTEM CONFIGURATION}

\section{A. System Concept}

Once the information of tag that patient possesses through a reader, the information of tag is transmitted to Atmega128 which is an MCU. In order to send the information on the tag, Atmega128 gets to transmit patient's information with a $\mathrm{PC}$ at a remote distance through a W3100A chip providing TCP/IP environment.

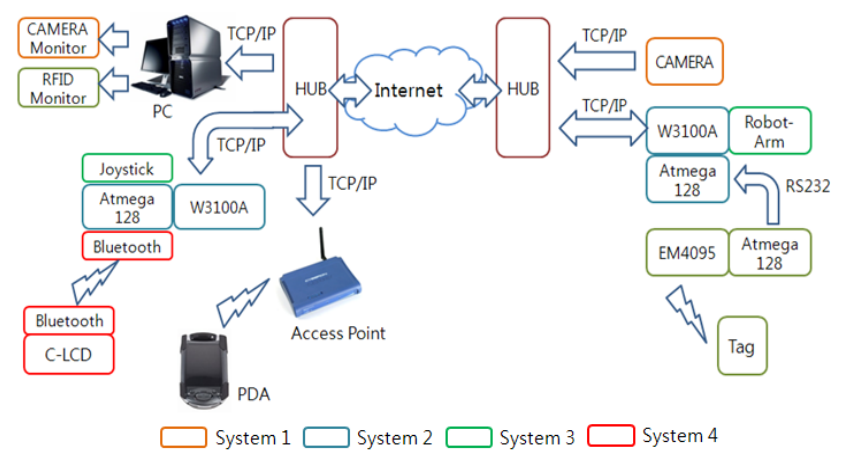

Figure1. Block diagram of overall System

In Figure 1 , the overall $\mathrm{H} / \mathrm{W}$ configuration can be classified into 4 systems. The first system is the RFID system of $125 \mathrm{KHz}$ and reads ID value of the tag. The RFID system is consisted of Reader, RF tag and antenna. The second system is a communication system for using TCP/IP communication and used a W3100A chip. TCP/IP is a standardized communication protocol enabling communication between computers on the internet. The remote data transmission uses W3100A and Atmega128. The third system is a wireless communication system using Bluetooth. The data received through TCP/IP communication is transmitted to an MCU. Also, the data is transmitted by Bluetooth communication. The network configuration of Bluetooth is consisted of Master and Slave relationship. The used frequency is the ISM band of $2.4 \mathrm{GHz}$ that can be used without authorization. The 
reliability of this communication is that a stable communication is possible in an environment with a lot of noise using frequency hopping method (FHSS). The fourth system is the wireless communication between $\mathrm{PC}$ and PDA. The communication between PC and PDA has used WLAN technology. The robot's arm was controlled by 3 server motors using joystick and 2 buttons. The server motor has used AX-12 motor enabling easy manufacture and control of robot's arm while Atmega128 was used for motor control.

\section{B. Hardware Composition}

\section{1) RFID System}

In RFID system, the EM4095 of SWATCH GROUP was used for the reader [17].

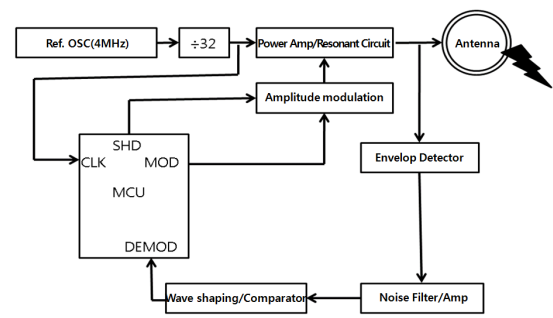

Figure2. Block Diagram of $125 \mathrm{KHz}$ RFID Reader

EM4095 is a CMOS all purpose device using $125 \mathrm{KHz}$ frequency band. The tag information is transmitted to tag antenna. The information on the tag is read through antenna of reader. The EM4100 which is in a form of a card has been used for the tag and this tag operates as Read Only. The reader system was made to operate as Read Only Mode and the tag ID value read by the reader is transmitted to MCU through RS232 communication.

\section{2) Ethernet Communication System}

The MCU for Ethernet communication has used Atmega128. ATmega128 is an RISC structure developed by Atmel Company and low power CMOS 8 bit micro controller [15]. The W3100A of WIZNET Company was used for TCP/IP communication [13].

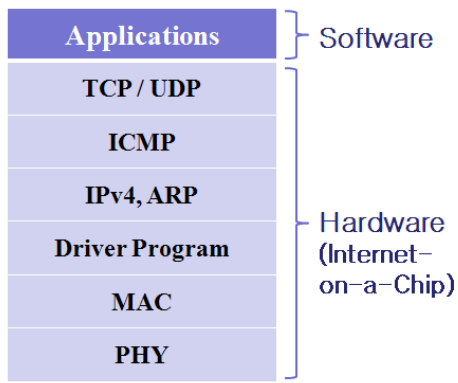

Figure3. Hardwired Stack of W3100A

Figure3 shows Hardwired Stack structure of W3100A. W3100A is LAN LSI consisted of an element using OS Kernel and Chip (Hardware logic) implementing MAC and PHY aspects. An additional RTL8201 chip is required for TCP/IP communication. That's because RTL8201 chip supports the Ethernet physical layer.

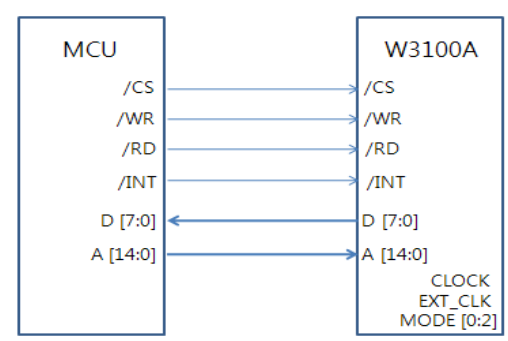

Figure4. W3100A Direct Address Mode

Figure 4 shows connection status for a direct control between W3100A and MCU. Direct Address Mode deals with all Registers of W3100A and Atmega128 on 1:1 basis at the Address Space. In Figure 4, the pin connected between W3100A and MCU (/CS, /WR, /RD, /INT) must be logic "Low" to operate.

\section{3) Control System for Robot-Arm}

a) Stick Controller

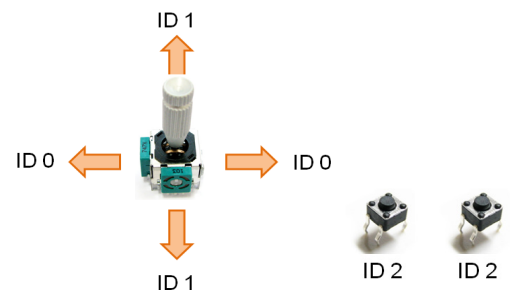

Figure 5 Control unit by ID value of AX-12

Inside the Stick Controller in Figure5 (Left), a variable resistance indicating movement in horizontal and vertical direction is attached. The voltage drop occurs from variation of variable resistance value and the location of stick can be found out with this voltage drop. In other words, the location of stick user has controlled can be found out with the voltage of both ends of variable resistance. The stick control is possible using two AX-12 motors used in the system. The horizontal axis can be controlled by motor AX-12 of ID 0 and the vertical axis can be controlled by motor AX-12 of ID 1 . The motor AX-12 of ID 3 can be controlled with two buttons in Figure5 (right).

\section{b) Robot-Arm}

AX-12 has been used for the operation of Robot-Arm. AX-12 is a Module type where Motor, Motor Driver, Control Unit and even Network are formed as one unit which is a Smart Actuator. AX-12. The motor inside AX12 is able to create a great torque although it is Compact size and made with special material that can endure strong external force. 


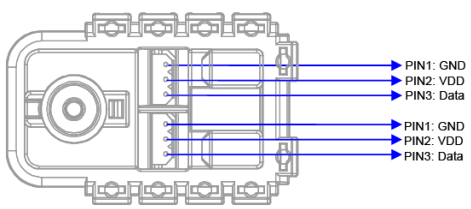

Figure6 AX-12 Pin Assignment

AX-12's can be used by connecting in series. The rotation angle of inner $\mathrm{AX}-12$ motor is $360^{\circ}$ and the input voltage for operation is $7 \mathrm{~V} \sim 10 \mathrm{~V}$. And ID value can be assigned for each motor and can be set up on a total of 254 motors from 0 to 253. The pin arrangement of AX-12 motor is shown as Figure 5. The Main Controller to drive AX-12 must support Half Duplex UART of TTL level. In this paper, Atmega128 was used as Main Controller and the communication speed is $9600 \mathrm{bps}$.

\section{4) Camera Monitor System}

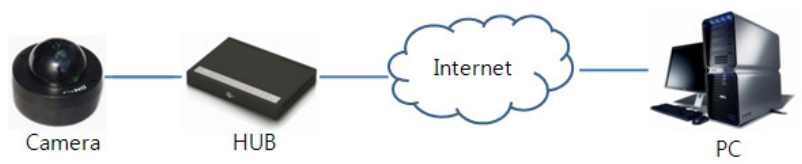

Figure7 Data flow of Network Camera

In order to monitor the treatment progress, a network camera is used. This camera in Fig. 7 can be monitored even without PC. The DMC-0101 model of DMZERO1 Company has been used for the camera. A 32bit ARM9 processor was used and connected to network by getting the IP assigned. The screen can be viewed using ID and Password on the Web browser for the monitoring. The IP and detailed settings can be set up by entering the IP address of network camera without a separate program. In case of using recording function, a low capacity image file of about $1 \mathrm{MB}$ per minute is made.

\section{5) Bluetooth System}

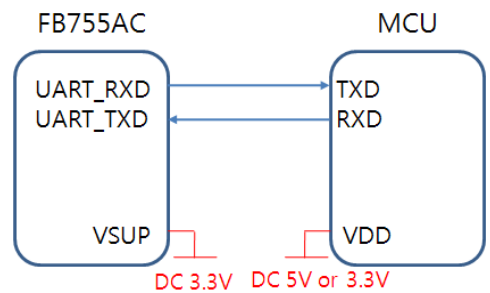

Figure8 Interface between FB755ACand MCU

The FB755AC model of Firmtech Company was used for Bluetooth [18]. FB755AC uses UART communication of Atmega128 and enables wireless communication in Fig. 8. The operation voltage of Atmega128 is 5V. But FB755AC operates with voltage of $3.3 \mathrm{~V}$. And the voltage was lowered to $3.3 \mathrm{~V}$ using diode. FB755AC is able to communicate with miniature terminal units that are able to display C-LCD using wireless communication with another Bluetooth through ISM band.

\section{Operating $S / W$}

1) $T C P / I P$ System

RFID Reader reads in the tag ID value of 10Byte through an antenna. EM4095 transmits to MCU when tag ID value is received using Interrupt of Atmega128.

TABLE1.

\section{PROGRAM FOR DATA TRANSMISSION IN MCU}

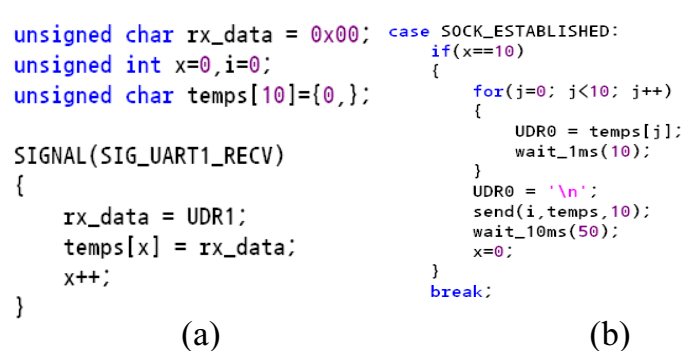

(a)

(b)

Atmega128 saves tag ID value through an arrangement like (a) source of Table 1. And the data is transmitted to remote distance by sending data to W3100A. Table1 (b) shows the details on the part where the value saved in the arrangement is sent to remote distance.

Atmega128 uses two USART enabling full duplex communication. UDR0 is used for RS232 communication with PC and UDR1 is used for Bluetooth communication. Table 2 (a) is the source and the delay of $1 \mathrm{~ms}$ is used each time one text is sent to transmit data in a stable way. recv ptr is a data pointer value received through TCP/IP.

\section{2) Bluetooth Operating Program}

The data to be transmitted to remote distance is sent to Atmega128 first and uses two UART enabling full duplex communication. UDR0 is used for RS232 communication with PC and UDR1 is used for Bluetooth communication [11]. The program for these to communication is Table 2 (a). recv ptr is a data pointer value received through TCP/IP. Bluetooth sends the tag information wireless with receiver Bluetooth at a remote distance. And the receiver Bluetooth shows tag information with CLCD. The source transmitting data with Bluetooth through UART is shown as Table2 (b).

TABLE2.

PROGRAM FOR BLUETOOTH COMMUNICATION IN PC USING UART

\begin{tabular}{|c|c|}
\hline 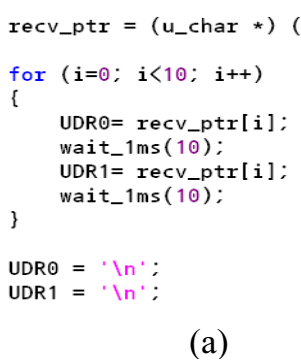 & $\begin{array}{l}\text { (1) } \\
\text { if }(x==10) \\
\quad \text { for }(i=0 ; i<10 ; i++) \\
\{\quad \text { UDR0=temps }[i] ; \\
\text { delay_ms }(10) ; \\
\text { UDRO }=\text { 'In'; } \\
x=0 ; \\
\text { (b) }\end{array}$ \\
\hline
\end{tabular}


The PC compares the value received through RS232 communication to display information of patient on the monitor. Once the PC and PDA receive data after connecting through TCP/IP, the data is transmitted to PDA. The data received on PDA is compared with the tag value registered in the PDA. The user information is display on the screen if there is matching value.

\section{3) Control Program of Robot-Arm}

a) Stick Controller

Stick Controller detects movement through a voltage value of variable resistance connected to the stick. The ADC (Analog Digital Convertor) was used in order to show the voltage value. Atmega128 has 8 channels of $\mathrm{ACD}$ with 10 bit resolving power and connected to ADC by multiplexer [14]. The ADC conversion result is saved to 16 bit data register by being arranged to the left or right.

TABLE3.

PROGRAM FOR STICK CONTROL USING ADC

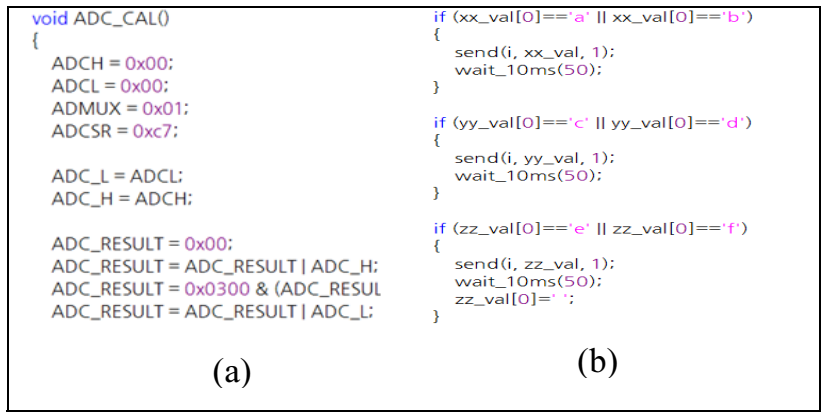

Table 3 (a) shows the source to initialize the upper/lower value of $\mathrm{ADC}$ and save the $\mathrm{ADC}$ conversion result value to a variable. Among ADC related registers, ADMUX designates the selection of ADC analog input channel, selection of conversion standard voltage source and saving format of conversion results. $\mathrm{ADCH}$ and $\mathrm{ADCL}$ are the registers saving $\mathrm{A} / \mathrm{D}$ converted results and have different saving format depending on whether ADLAR bit value of ADMUX register is ' 0 ' or ' 1 '. In Table 3 (b), the source transmitting the value of $\mathrm{X}$ axis, $\mathrm{Y}$ axis and $\mathrm{Z}$ axis through TCP/IP according to the movement of Stick is shown.

\section{b) Robot-Arm}

MCU and AX-12 communicate by exchanging packets. For the types of packets, there are Instruction Packet transmitted from MCU to AX-12 and Status Packet transmitted from AX-12 to MCU. Instruction Packet is a Packet where MCU instructs movement to AX-12.

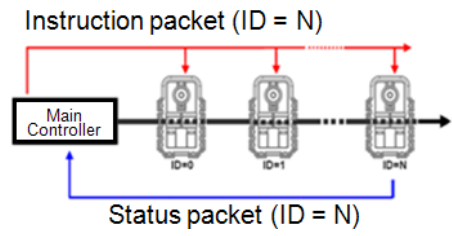

Figure9 Communication between $\mathrm{AX}-12$ and $\mathrm{MCU}$
In case the MCU sends Instruction Packet where MCU is set as $\mathrm{ID}=\mathrm{N}$ at a system where many $\mathrm{AX}-12$ 's are connected to one MCU, only the AX-12 with ID of N among many AX-12's return the Status Packet and performs that Instruction.

TABLE4.

PROGRAM FOR DATA TRANSMISSION IN MCU

\begin{tabular}{|c|c|}
\hline & \\
\hline MCU_initialize(void) & void AX_12_initialize(void) \\
\hline outp (OxFF, DDRA); & $\begin{array}{l}\text { PORTE = } 0 \times 01 ; \\
\text { DDRE }=0 \times 01:\end{array}$ \\
\hline RIA): & UCSR1A $=0 \times 02$ \\
\hline outp (OxFF, DDRB); & $\begin{array}{l}\text { UCSR1B }=0 \times 08 \\
\text { UCSR1C }=0 \times 06\end{array}$ \\
\hline (U) & UBRR1H = ON00; \\
\hline outp (OxOf, DDRE); & UBRR1L = $0 \times 01$ \\
\hline outp(0x00, PORTE); & Delay_ms $(200)$ : \\
\hline & \\
\hline (a) & (b) \\
\hline
\end{tabular}

The Protocol of AX-12 uses 8 bit, 1 Stop bit and Asynchronous Serial communication of None Parity. Status Packet is a packet returned to MCA as response by AX-12 after receiving the Instruction Packet.

For initialization functions to operate AX-12, there are MCU initialize function and AX 12 initialize function. Table 4(a) shows MCU_initialize and sets up the pin of MCU as well as TXD and RXD pins.

AX-12_initialize is the process of initializing the AX-12+ motor, and used for settings to control AX-12 such as the setting the Baud Rate of USART, transmission/ reception activation and data size or parity mode, etc. Table 4 (b) shows the AX-12 initialize function while the busy ratio of UART1 clock was set as 16 and data bit was set as 8 bit.

\section{4) Operating Program of RFID}

EM4095 Chip can be made as Read Only Mode or Read/Write Mode by surrounding circuit. Read Only Mode connects the MOD pin of EM 4095 to GND and only performs demodulation without modulation function [1][2][3]. Read/Write Mode transmits modulated data with MOD pin by connecting the MOD pin to ATmega128. In order to average the cycle value of digital signal of the data coming out from No. 13 pin which is the data output section of EM4095 Chip, the algorithm was implemented using Interrupt of ATmega12 8 to create $\mathrm{x}$ value as $30<\mathrm{x}<50$ and $65<\mathrm{x}<85$. MOD executes $100 \%$ modulation once connected to VOD and the antenna current is defined as the numerical expression Eq. (1).

$$
I_{a n t}=\frac{4}{\pi} \frac{V D D-V S S}{R_{a n t}+R_{s e r}+2 R_{a d}}
$$

\section{EXPERIMENT AND PERFORMANCE}

Once the tag of patient is read through RFID Reader, the tag ID value is saved through Atmega128. Atmega128 enables data transmission to a remote PC through W3100A 
providing TCP/IP environment. The data received from a remote distance is transmitted to Bluetooth, PC and finally up to PDA. The PC displays patient information by comparing the received data with $\mathrm{DB}$ data. The treatment is possible by checking the information of patient and controlling robot's arm of remote distance using joystick and buttons. And the control is enabled by checking the screen on real time basis through a web camera. Figure 10 is the configuration of overall system showing transmitter and receiver. The transmitter is configures as $125 \mathrm{KHz}$ RFID system, W3100A, robot's arm and web camera. The receiver is configured as W3100A, Bluetooth, PDA and Joystick.

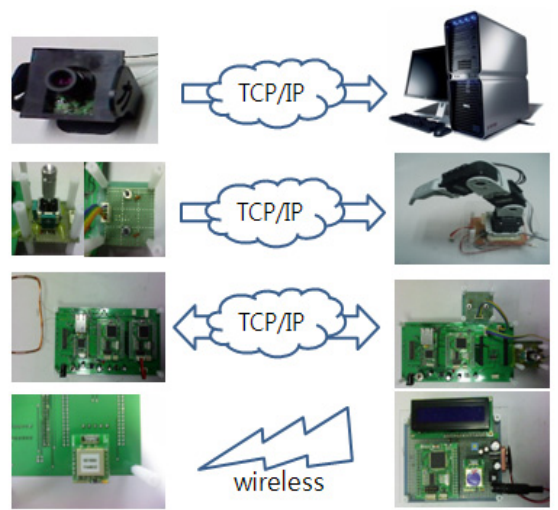

Figure 10. Communication Method on each Part(See Table5)

Table 5 shows the communication method on each part of the system, response speed of data and error margin. The 2 Computers was used for measurement. The error margin is very low in general and created operation delay only at a transmission using joystick. This problem would be solved using precision joystick.

TABLE 5.

\section{PERFORMANCE OF COMMUNICATION EACH PART}

\begin{tabular}{|c|c|c|}
\hline System Part & $\begin{array}{c}\text { Communicati } \\
\text { on Method }\end{array}$ & $\begin{array}{c}\text { Response Time/ } \\
\text { Error Rate }\end{array}$ \\
\hline $\begin{array}{c}\text { Network Camera } \\
\text { ((a) in Fig.10) }\end{array}$ & TCP/IP & $0.05 \mathrm{Sec} / 0 \%$ \\
\hline $\begin{array}{c}\text { Stick Control } \\
((\mathrm{b}) \text { in Fig.10) }\end{array}$ & $\mathrm{TCP} / \mathrm{IP}$ & $\begin{array}{c}0.5 \mathrm{sec} / \\
1.5 \%(\text { in Local Area) }\end{array}$ \\
\hline $\begin{array}{c}\text { RFID } \\
((\mathrm{c}) \text { in Fig.10) }\end{array}$ & $\mathrm{TCP} / \mathrm{IP}$ & $0.09 \mathrm{sec} / 0 \%$ \\
\hline $\begin{array}{c}\text { PDA, CLCD } \\
((\mathrm{d}) \text { in Fig.10) }\end{array}$ & Bluetooth & $\begin{array}{c}0.08 \mathrm{sec} / \\
0 \%(\text { in Local Area })\end{array}$ \\
\hline
\end{tabular}

\section{CONCLUSION}

The overall system was configured as $125 \mathrm{KHz}$ RFID, TCP/IP, Bluetooth, PDA, robot's arm and joystick. The $125 \mathrm{KHz}$ RFID system transmits tag information to MCU. The Atmega128 which is an MCU transmits tag information to $\mathrm{W} 3100 \mathrm{~A}$ and a remote data transmission is enabled through TCP/IP communication. And Atmega128 is able to transmit data to $\mathrm{PC}$ and Bluetooth through
UART. Bluetooth enables remote communication using wireless communication of ISM band. The receiver Bluetooth displays tag information on CLCD. The PC compares the received tag information to display the patient information saved in the DB on the monitor.

And the TCP/IP communication is possible between PC and PDA. PDA is able to receive the data of PC by wireless communication through AP. It can also control the robot's arm at a remote distance using joystick and buttons. The movement can be seen through PC using web camera in order to check the movement of robot's arm. This system can be useful in a field where remote control is required by having an advantage of being able to control from a remote area.

\section{REFERENCES}

[1] H. K. Jo, "Production Manual of RFID system using U2270B", Hong-Neng Publish, 2008, ISBN978-89-7283-552-3

[2] H. J. Lee, H. K. Jo, "Security and System of RFID", Com-One Media. 2009, ISBN 978-89-92475-25-9

[3] H.K. Jo, H.J. Lee, "A Relay Transmission of the RFID Tag ID over the Wireless and TCP/IP with Security Agent" LNAI, Springer Verlag, 2007. 918-927

[4] Y.H Kim, H.K. Jo, "Patient Information Display System in Hospital Using RFID”, ICHIT2009, 2009, pp. 397-400

[5] William Stalling, "Data and Computer Communications", Prentice Hall, The $8^{\text {th }}$ Edition, 2007

[6] Behrouz A, Forouzan, "TCP/IP Protocol Suite, McGraw Hill, the $3^{\text {th }}$ Edition, 2007

[7] Leon Garcia, Widjaja, "Communication Network : Fundamental Concepts and Architectures", McGraw Hill, 2001

[8] J.G Choi, "Visual C\#.NET 2005 2nd Edition Project", Young-Jin Publish, 2007.

[9] I.G. Kim, G.S. Kim, S.J. Ha, Y.J. Kim,”, "Programming of C\#.NET”, Inter-vision, 2007

[10] S.U. Kim, "Window Network Programming TCP/IP Socket Programming", Han-Bit Media, 2007

[11] D.U. Shin, C.H. Oh, "Easy-Learning of AVR ATmeg a128", Ohm$\mathrm{Sa}, 2004$

[12] Y.J. Sung, J.G. Choi, "Practice Project of Visual C\#.NET 2003", Yung-Jin Publish, 2005

[13] S.U. Yuan, "TCP/IP Socket Programming”, Free-Lack, 2004

[14] D.B. Jin, "Application of The Atmega128", Yang-Se Gack, 2004

[15] Institute Technology Micom World, "Theory and Experiment Using AVR (ATmega128)", Micom-World, 2004

[16] http://www.atmel.com/avr

[17] http://www.emmicroelectronic.com

[18] http://www.Firmtech.co.kr

[19] http://www.realtek.com.tw

[20] http://www.wiznet.co.kr

[21] http://www.micomworld.co.kr

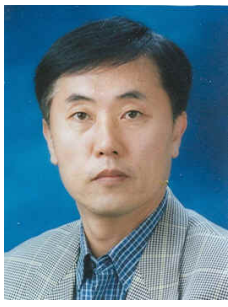

Heung-Kuk Jo Birthday: 02, December, 1955. I received the B.C. and M.S. Degree in Wireless Communication from Dong-A University, BUSAN, KOREA, in 1977 and 1979, respectively. I received Dr.-Ing.(PhD) Degree in Electrical Acoustic from Technical University Berlin, Germany in 1989. I am a Professor of the Department of Computer Information Engineering of DONG-SEO University from March 1992 to present. My areas of research include wireless communication and RFID system. I had a Industry Experience at SAMSUNG Electronics in SUWOON from February 1990 to February 1992. E-Mail: hkjo@gdsu.dongseo.ac.kr 